\title{
Effects of Neoadjuvant Radiotherapy on Postoperative Complications in Rectal Cancer: A Meta-Analysis
}

\author{
Jianguo Yang, ${ }^{1}$ Yajun Luo, ${ }^{1}$ Tingting Tian, ${ }^{2}$ Peng Dong $\left(\mathbb{D}^{1},{ }^{1}\right.$ and Zhongxue Fu ${ }^{1}{ }^{1}$ \\ ${ }^{1}$ Department of Gastrointestinal Surgery, The First Affiliated Hospital of Chongqing Medical University, \\ Chongqing 400016, China \\ ${ }^{2}$ Department of Gastroenterology, Zhutuo Town Health Center of Chongqing, Chongqing 402191, China \\ Correspondence should be addressed to Peng Dong; carry_dong@126.com and Zhongxue Fu; fuzhongxuecq@163.com
}

Received 12 August 2021; Revised 29 November 2021; Accepted 18 December 2021; Published 5 January 2022

Academic Editor: Irena Ilic

Copyright (c) 2022 Jianguo Yang et al. This is an open access article distributed under the Creative Commons Attribution License, which permits unrestricted use, distribution, and reproduction in any medium, provided the original work is properly cited.

\begin{abstract}
Objective. Neoadjuvant radiotherapy (nRT) is an important treatment approach for rectal cancer. The relationship, however, between nRT and postoperative complications is still controversial. Here, we conducted a meta-analysis to evaluate such concerns. Methods. The electronic literature from 1983 to 2021 was searched in PubMed, Embase, and Web of Science. Postoperative complications after nRT were included in the meta-analysis. The pooled odds ratio (OR) was calculated by the random-effects model. Statistical analysis was conducted by Review Manager 5.3 and STATA 14. Results. A total of 23,723 patients from 49 studies were included in the meta-analysis. The pooled results showed that nRT increased the risk of anastomotic leakage (AL) compared to upfront surgery $(\mathrm{OR}=1.23 ; 95 \% \mathrm{CI}, 1.07-1.41 ; p=0.004)$. Subgroup analysis suggested that both long-course $(\mathrm{OR}=1.20,95 \%$ CI $1.03-1.40 ; p=0.02)$ and short-course radiotherapy $(\mathrm{OR}=1.25,95 \% \mathrm{CI}, 1.02-1.53 ; p=0.04)$ increased the incidence of AL. In addition, nRT was the main risk factor for wound infection and pelvic abscess. The pooled data in randomized controlled trials, however, indicated that $\mathrm{nRT}$ was not associated with $\mathrm{AL}(\mathrm{OR}=1.01 ; 95 \% \mathrm{CI} 0.82-1.26 ; p=0.91)$. Conclusions. nRT may increase the risk of $\mathrm{AL}$, wound infection, and pelvic abscess compared to upfront surgery among patients with rectal cancer.
\end{abstract}

\section{Introduction}

Colorectal cancer (CRC) is a common malignant tumor globally that is ranked third in terms of incidence and second in terms of mortality. It is estimated that over 1.8 million new cases of CRC occur each year [1]. In the past few decades, the development of total mesorectal excision (TME) has greatly improved the oncological outcome of rectal cancer patients. However, local recurrence, distant metastasis, and chemoradiotherapy resistance are still the main causes of death in rectal cancer patients [2,3]. Studies have shown that preoperative chemoradiotherapy downstages the primary tumor, increases the possibility of radical resection, increases the sphincter-preserving rate, and reduces the risk of local recurrence of rectal cancer [4-7]. Therefore, TME after neoadjuvant chemoradiotherapy (nCRT) has become the standard treatment for locally advanced rectal cancer. After nCRT or total neoadjuvant therapy (TNT), approximately $15-30 \%$ of rectal cancer patients can achieve pathological complete response (PCR), which significantly improves the oncological outcome of patients [8-10].

Postoperative complications are closely related to local recurrence and distant metastasis of rectal cancer [11]. AL is one of the common postoperative complications of rectal cancer. It has been reported that $\mathrm{AL}$ increases the risk of systemic, peritoneal, and local recurrence of CRC $[12,13]$, and the possible mechanism is that the inflammatory reaction results in an increase in proinflammatory and proangiogenic factors, which may stimulate the growth of residual tumor cells. In addition, inflammation caused by $\mathrm{AL}$ contributes to tumor escape immune surveillance by suppressing $\mathrm{T}$ cell $[14,15]$. Several meta-analyses have shown that AL decreases overall survival and disease-free survival and increases the risk of cancer-related death in rectal cancer patients [16-18]. Many factors may affect postoperative complications, including age, sex, tumor location, and 
diabetes mellitus [19-21]. Some studies have reported that chemoradiotherapy may create local rectal tissue injury and influence anastomosis healing. However, it remains controversial whether preoperative chemoradiotherapy leads to an increase in complications after rectal cancer surgery [22-24].

We conducted the present meta-analysis to explore whether nRT increases the risk of postoperative complications for rectal cancer. The primary outcome of interest was $\mathrm{AL}$, and the secondary outcomes of interest included wound infection, pelvic abscess, urinary tract infection, ileus, hemorrhage, reoperation, overall complications, and mortality.

\section{Methods}

The systematic review and meta-analysis were performed based on the preferred reporting items for the systematic review and meta-analysis 2020 statement (PRISMA 2020 statement) [25] (Table S1).

\subsection{Literature Search Strategy. PubMed, EMBASE, and Web} of Science electronic databases were searched by two authors (Yang and Luo) with the following subject terms: (rectal cancer) OR (rectal neoplasms); (neoadjuvant therapy) OR (neoadjuvant radiotherapy) OR (neoadjuvant chemoradiotherapy) OR (perioperative therapy) OR (perioperative radiotherapy) OR (perioperative chemoradiotherapy); and (complications) OR (morbidity) OR (anastomotic leakage) OR (anastomotic leak). We combined the search items using "AND". The last data retrieval was June 1, 2021, and language restrictions were not considered. The reference lists of selected articles were searched to find potentially relevant studies. Titles, abstracts, and full texts of studies were accessed to exclude inappropriate research. When several articles were published in the same cohort, only the latest publications were included. If there were inconsistent decisions, they were resolved by two reviewers through consultation. Otherwise, the final decision was made by a third reviewer $(\mathrm{Fu})$.

2.2. Study Selection Criteria. The inclusion criteria were as follows: (1) cohort studies and randomized clinical trials (RCTs); (2) English publication studies; (3) postoperative complications in the nRT group and upfront surgery group were compared; and (4) AL must be reported in eligible studies. The exclusion criteria were as follows: (1) reviews, letters, expert opinions, comments, case reports, and metaanalysis; (2) incomplete data (no primary outcome or detailed data of postoperative complications) or no full text; (3) nonhuman studies; and (4) nonrelevant literature.

2.3. Data Extraction and Quality Assessment. All data were extracted from full texts by two authors (Yang and Tian) with a standard spreadsheet. The collection information was as follows: (1) first author name, journal name, publication time, nation, and the number of participants; (2) basic characteristics and therapy process of rectal cancer patients; and (3) postoperative complications (AL, wound infection, pelvic abscess, urinary tract infection, ileus, hemorrhage, reoperation, and overall complications) and mortality.

The quality of cohort studies was evaluated independently according to the Newcastle-Ottawa Scale (NOS) by two reviewers (Luo and Peng). The NOS Scale of cohort study included study population option, comparability, and exposure or outcome assessment. The maximum score was 9 points for cohort studies, and studies with a score of 6 or more were considered high quality [26]. Two researchers used the Cochrane risk-of-bias tool (RoB 2.0) to independently assess the quality of randomized controlled trials. The study was assigned an overall score: "low," "some concerns," or "high." [27]. Any disagreement issues were resolved through negotiating with each other or consulting with a third reviewer.

2.3.1. Definition. The diagnosis of AL was required to meet at least one of the following conditions: (1) intestinal contents and/or gas leakage into the abdomen or pelvis from the anastomotic site and extravasated through the wound, drainage tube, or anus; (2) postoperative recurrent fever, abdominal pain, sepsis, peritonitis and/or organ failure; and (3) confirmed by imaging examination (such as $\mathrm{X}$-ray, endoscopy, computed tomography, magnetic resonance imaging, or ultrasound) or digital rectal examination or surgery [28]. AL that was only detected by imaging but had no clinical manifestations was defined as "asymptomatic" AL. We only pooled symptomatic AL data in the present meta-analysis. Wound infection included abdominal and perineal wound infection. Overall complications included surgical and nonsurgical complications within 30 days after surgery. Mortality was defined as death within 30 days after surgery or during hospitalization. Neoadjuvant radiotherapy (nRT) included short-course radiotherapy (SRT), long-course radiotherapy (LRT), and chemoradiotherapy (CRT).

2.4. Statistical Analysis. Statistical analysis was conducted by Review Manager version 5.3 (The Nordic Cochrane Center, The Cochrane Collaboration, Copenhagen, Denmark) and STATA 14 (Stata Corporation, College Station, TX, USA). Dichotomous data were summarized as odds ratios (ORs) with 95\% confidence intervals (CIs) using the Mantel-Haenszel method [29]. Due to the expected heterogeneity between studies, the random-effects model was applied to all outcomes [30]. Heterogeneity was evaluated by the $\mathrm{I}^{2}$ index and $Q$ test. $\mathrm{I}^{2}$ values of $<25 \%, 25-50 \%, 50-75 \%$, and $75-100 \%$ suggest low, moderate, high, and extreme heterogeneity, respectively [29]. $I^{2} \geq 50 \%$ or $p<0.01$ (Cochran's $Q$ test) was considered significant heterogeneity. If there was significant heterogeneity in the pooled data, subgroup analysis was used to assess the potential reason for the heterogeneity [31]. Publication bias was evaluated by visual inspection of the funnel plot and Egger's test, providing that more than 10 studies with the primary outcome of interest were included [32]. The pooled OR and 95\% CI were 
represented by the forest plot. $p$ values less than 0.05 were considered significant.

In addition, we conducted a subgroup analysis of anastomotic leakage. The purpose was to explore the effect of neoadjuvant long-course radiotherapy, short-course radiotherapy, and the operation interval ( $<8$ weeks) after longcourse radiotherapy on anastomotic leakage. We also performed a subgroup analysis of RCT and non-RCT studies.

\section{Results}

3.1. Literature Search and Characteristics. According to the search strategy, 2854 articles were retrieved. After removing 960 duplicate documents, 1894 potentially related studies were included. A total of 1740 articles were excluded by reading the titles and abstracts. After reading the full text, the following 100 studies were excluded: results of primary interest not reported $(n=28)$; abstract, meta-analysis, reviews, case reports, and letters $(n=30)$; duplicate data from same patients $(n=6)$; non-English language text $(n=11)$; no full text $(n=1)$; neoadjuvant chemotherapy only $(n=16)$; others $(n=8)$. Finally, 10 RCTs, 1 prospective study, and 38 retrospective studies were included in the present metaanalysis (Figure 1).

In total, 23,723 individuals from 49 studies were included in the meta-analysis. Of these individuals, 12,082 received $\mathrm{nRT}$, and 19,502 underwent primary anastomosis after rectal cancer surgery. Eleven of the 49 studies reported SRT with a scheme of $5 \mathrm{~Gy}$ each time for 5 consecutive days. Radical operation was conducted within 1 week after the completion of SRT. The majority of CRT was combined with fluorouracil-based concurrent chemotherapy, and the concurrent chemotherapy regimens mainly included 5FU, $5 \mathrm{FU}+\mathrm{LV}$, and capecitabine. The specific characteristics of each study are shown in Table 1 and Table S2.

\subsection{Assessment of Methodological Quality and Validity.} The quality of the cohort study was assessed by the NOS scale. The results showed that the bias of the included studies was acceptable, because all studies received a score of five stars or above. We assessed the risks of bias of the 10 included RCTs using Cochrane RoB 2.0. Six RCTs were considered to be at low risk of bias. Four RCTs had some concerns because of the randomization process or deviation from the intended intervention (Table S3 and Figure S7).

3.3. Anastomotic Leakage. For the primary endpoint of AL, 49 studies were analyzed. A total of 19,502 patients underwent anterior resection, including 9919 patients from the nRT group. The pooled OR of AL was $1.23(95 \% \mathrm{CI}$, $1.07-1.41 ; p=0.004$ ) (Figure 2). There was low heterogeneity among these studies $\left(\chi^{2}=56.33, p=0.19 ; I^{2}=15 \%\right)$.

We conducted a subgroup analysis for AL. For one subgroup analysis, 39 studies reported the incidence of AL in patients with long-course radiotherapy (including CRT) before surgery. The pooled data showed that the risk of AL in patients with long-course radiotherapy was higher than that in patients without long-course radiotherapy $(\mathrm{OR}=1.20$,
95\% CI, 1.03-1.40; $p=0.02)$ and no obvious heterogeneity was detected $\left(\chi^{2}=41.60, p=0.32 ; I^{2}=9 \%\right)$ (Figure 3). Eleven studies of patients receiving SRT were included in the metaanalysis. The pooled data revealed that SRT also increased the incidence of $\mathrm{AL}(\mathrm{OR}=1.25,95 \% \mathrm{CI}, 1.02-1.53 ; p=0.04)$ with low heterogeneity $\left(\chi^{2}=11.30, p=0.33 ; I^{2}=11 \%\right)$ (Figure S1).

Ten RCTs involving 3951 patients with rectal cancer were assessed. The analysis demonstrated no significant association of AL with nRT (OR=1.01; 95\% CI 0.82-1.26; $p=0.91)$, and no heterogeneity was observed $\left(\chi^{2}=9.52\right.$, $\left.p=0.39 ; I^{2}=5 \%\right)$ (Figure 4$)$. In contrast, the pooled data in the 39 retrospective studies found that the incidence of AL in the nRT group was higher than that in the upfront surgery group $\quad(\mathrm{OR}=1.33 ; \quad 95 \% \quad$ CI $1.13-1.57 ; \quad p=0.0008)$ (Figure S2).

Twenty-eight studies reported that radical surgery was performed within 8 weeks after the completion of longcourse radiotherapy. The pooled results indicated that surgery within eight weeks did not increase the incidence of $\mathrm{AL}$ compared to the upfront surgery group $(\mathrm{OR}=1.12,95 \%$ CI, $0.94-1.34 ; p=0.20)$, and there was no significant heterogeneity among the included studies $\left(\chi^{2}=28.39, p=0.39\right.$; $I^{2}=5 \%$ ) (Figure S3).

3.4. Wound Infection and Pelvic Abscess. Wound infection data were analyzed in 25 studies, and 12 of these studies reported perineal wound infection after abdominoperineal resection. The pooled data showed that patients receiving nRT were more likely to suffer from wound infection $(\mathrm{OR}=1.42,95 \% \mathrm{CI}, 1.20-1.67 ; p<0.00001)$ (Figure 5). In addition, the risk of perineal wound infection after abdominoperineal resection in the nRT group was higher than that in the upfront surgery group $(\mathrm{OR}=2.14,95 \% \mathrm{CI}$, $1.72-2.68 ; p<0.00001)$, and the heterogeneity was not significantly different (Figure S4). For postoperative pelvic abscess, $\mathrm{nRT}$ showed a higher incidence of pelvic abscess than upfront surgery $(\mathrm{OR}=2.12,95 \% \mathrm{CI}, 1.52-2.96$; $p<0.00001$ ) (Figure S5).

3.5. Other Postoperative Complications. Ten studies reported urinary tract infections after surgery. The present study found that nRT did not increase the risk of urinary tract infections $(\mathrm{OR}=1.15,95 \% \mathrm{CI}, 0.77-1.71 ; p=0.41)$. Fourteen studies described postoperative hemorrhage, and the pooled incidence of postoperative hemorrhage in the nRT group and upfront surgery group was $2.83 \%$ and $2.78 \%$, respectively. In addition, nRT did not increase the incidence of postoperative ileus. The pooled data showed that the overall complications in the nRT group were higher than those in the upfront surgery group, but there was no significant difference $(\mathrm{OR}=1.15,95 \% \mathrm{CI}, 0.99-1.32, p=0.06)$. Moderate heterogeneity was observed in the meta-analysis $\left(\chi^{2}=37.47, p=0.06 ; I^{2}=39 \%\right)$. In this meta-analysis, the nRT group and the upfront surgery group had similar reoperation and mortality rates. The specific data on urinary tract infection, hemorrhage, ileus, overall complications, reoperation, and mortality are shown in Table 2. 


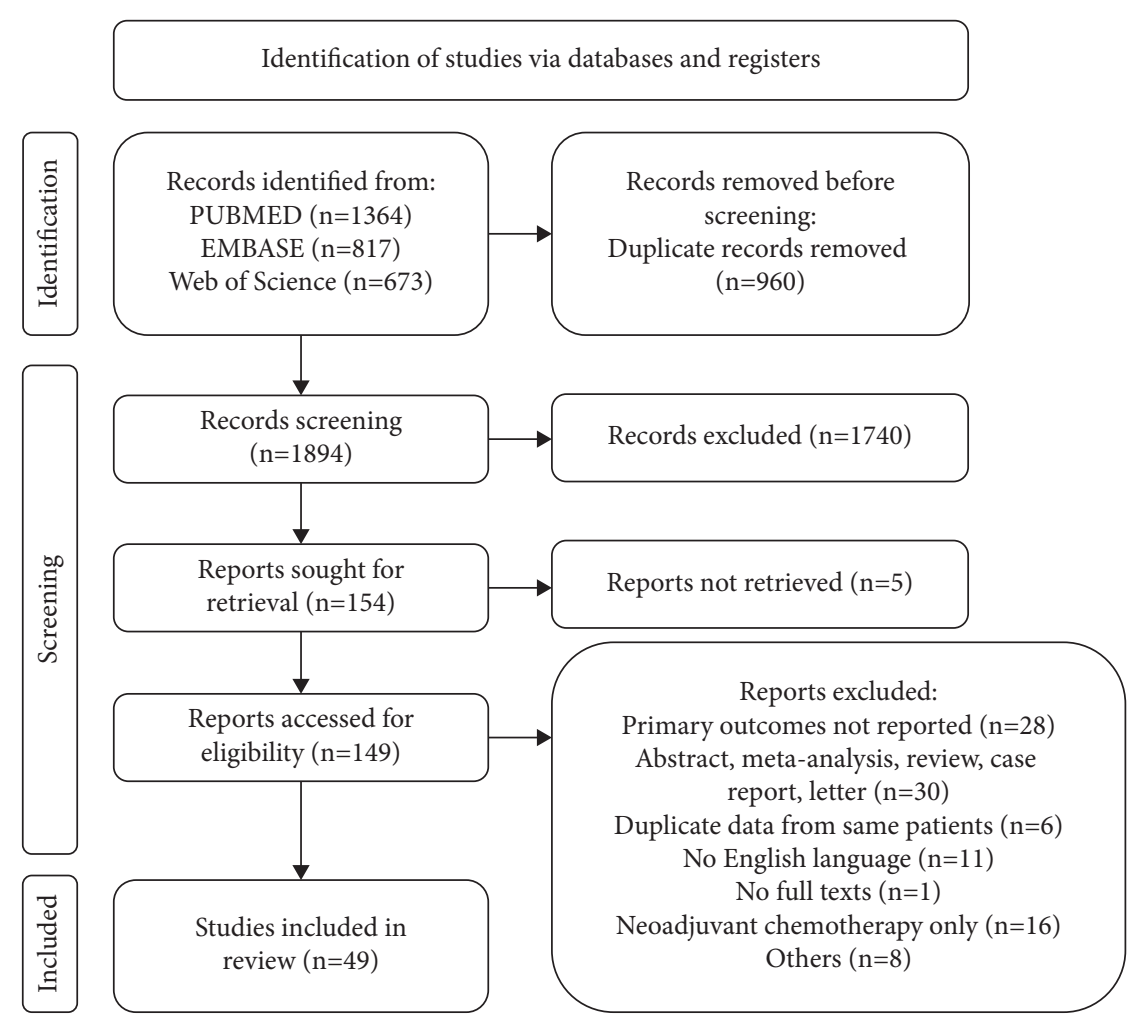

FIgURE 1: PRISMA flow diagram.

3.6. Publication Bias. The visual funnel plot distribution and Egger's test were used to assess the publication bias of AL. According to the funnel plot and Egger's test results, no publication bias was detected $(p=0.37$, Egger's test $)$ (Figure S6).

\section{Discussion}

The present meta-analysis was designed to investigate the effect of nRT on postoperative complications. The present study suggested that nRT may be related to increased incidence of AL compared to upfront surgery. Moreover, other anticipated results were as follows: nRT also increased the risk of postoperative wound infection and pelvic abscess.

In accordance with the present results, both short-course radiotherapy and long-term radiotherapy increased the incidence of AL within 30 days after surgery. Qin et al. [79] performed a randomized controlled trial and reported similar results to the present study. A total of 260 patients with anterior resection were enrolled in their study, of whom $61.92 \%$ received $\mathrm{nCRT}$. The incidence of symptomatic AL within 30 days after surgery was significantly higher than that of neoadjuvant chemotherapy alone (16.15\% vs. $5.1 \%)$. Additionally, Frouws et al. [46] retrospectively analyzed 3001 rectal cancer patients who underwent primary anastomosis, of whom 2211 patients received SRT. Their study found that the AL rate of Grades B and C in the SRT group was higher than the upfront surgery group $(10.58 \%$ vs. $7.59 \%)$. In contrast, there was no evidence that nRT was a risk factor for $\mathrm{AL}$ in multiple large randomized controlled trials. An earlier multicenter randomized controlled study in Sweden indicated that preoperative short-course radiotherapy did not increase the incidence of AL compared to surgery alone (10.97\% vs. $7.66 \%$ ) [63]. The trial conducted by Marijnen et al. [57] also found that the incidence of AL was similar between the short-course radiotherapy and surgery alone groups (11\% vs. 12\%). Another prospective study reported that short-course radiotherapy had a higher rate of AL than surgery alone (27.4\% vs. $20.6 \%)$, but the results were not significantly different [68].

Consistent with short-course radiotherapy, a phase III clinical trial performed in Korea showed that, compared with surgery alone, TME surgery after long-course radiotherapy did not result in more AL [65]. The CAO/ARO/ AIO-94 study reported that the incidence of AL was similar between the neoadjuvant chemoradiation group and adjuvant chemoradiation groups (11\% vs. $12 \%)$ [4]. Moreover, the meta-analysis including 10 RCTs also did find nRT would increase the occurrence of $\mathrm{AL}$ compared with upfront surgery. Considering that no association between AL and nRT was observed in RCTs, these results need to be interpreted with caution.

In the present meta-analysis, no evidence suggested that surgery within 8 weeks after completing radiotherapy would cause a higher risk of AL. The Lyon R90-01 study results indicated that prolonging the interval between completing radiotherapy and surgery may promote tumor downstaging, improve sphincter preservation, improve PCR rates, and have no influence on postoperative complications and oncological outcomes [80]. In recent years, an interval of 6-8 


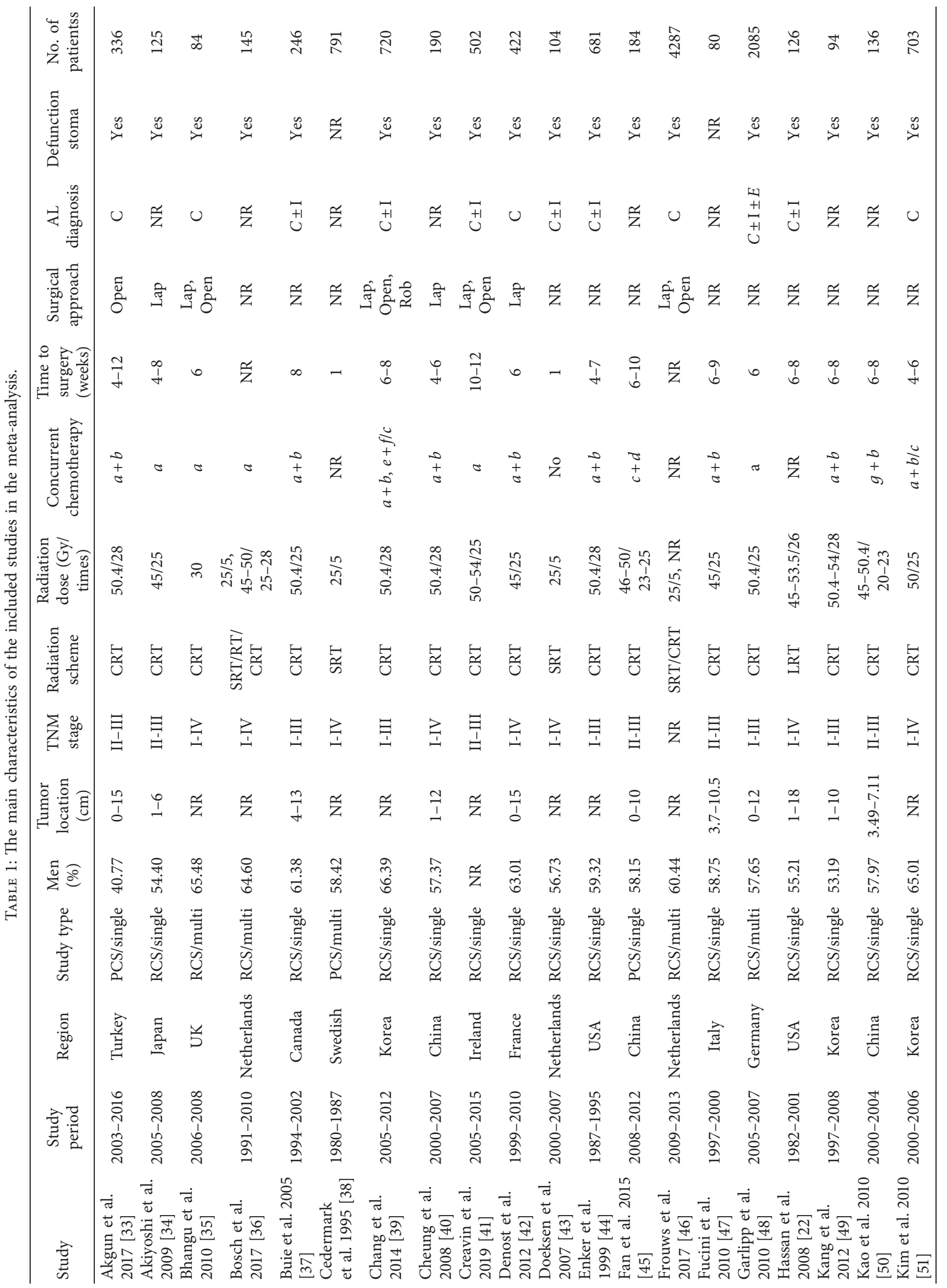




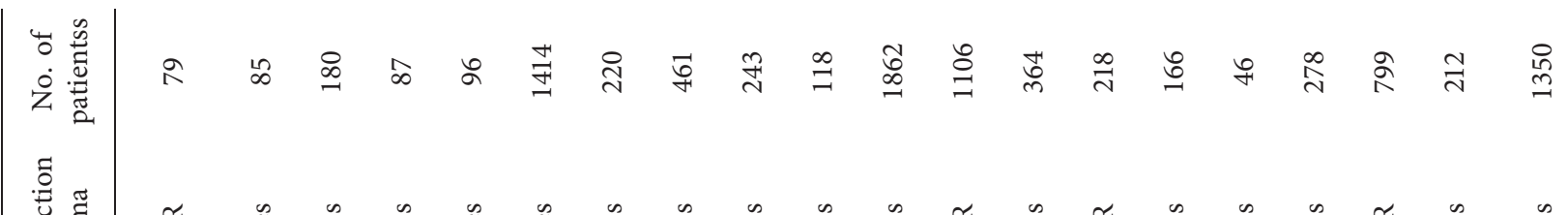

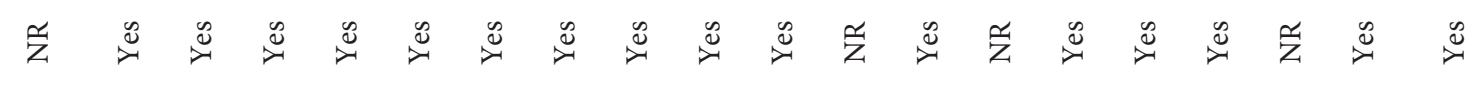
$\triangle$

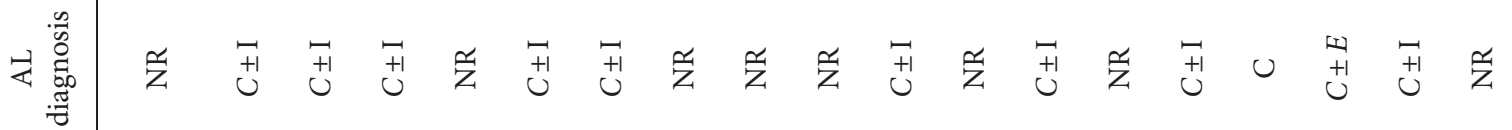

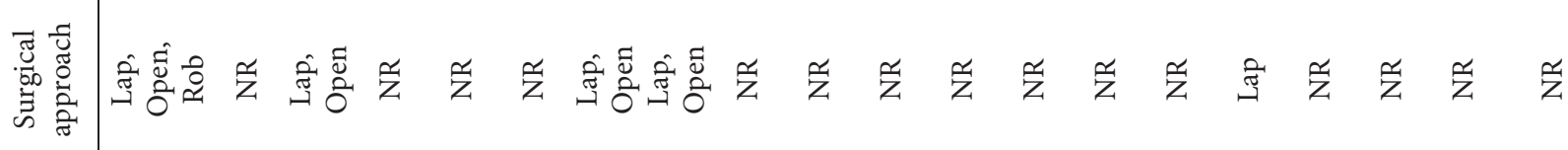

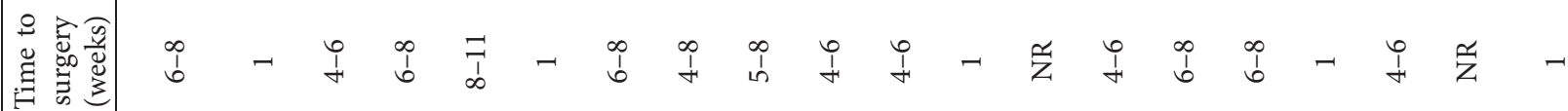

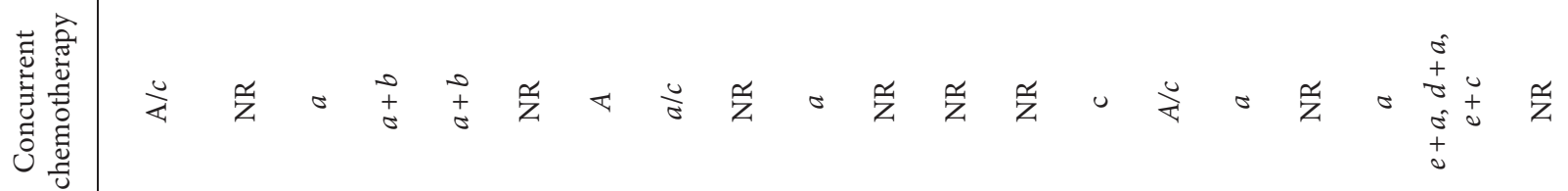

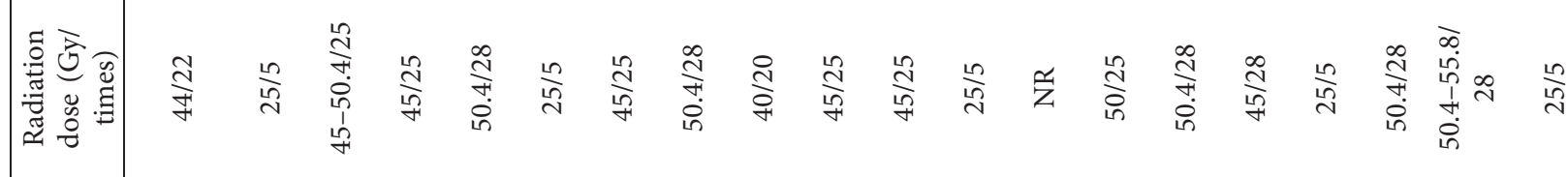

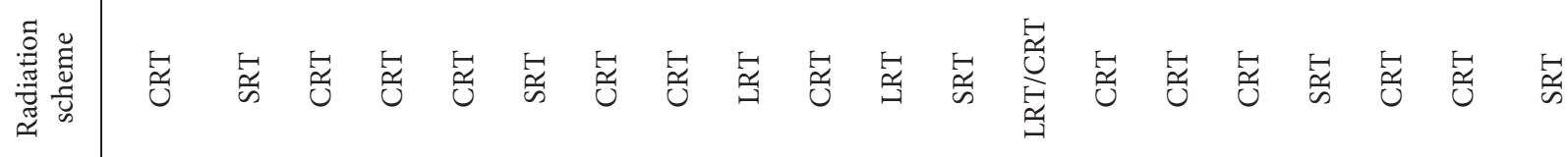

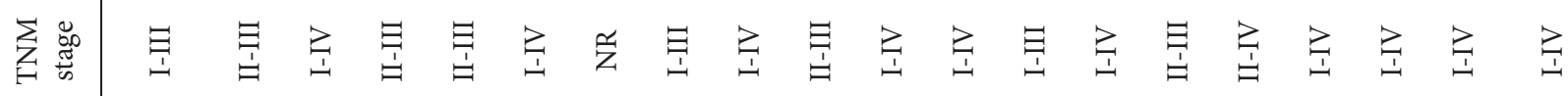

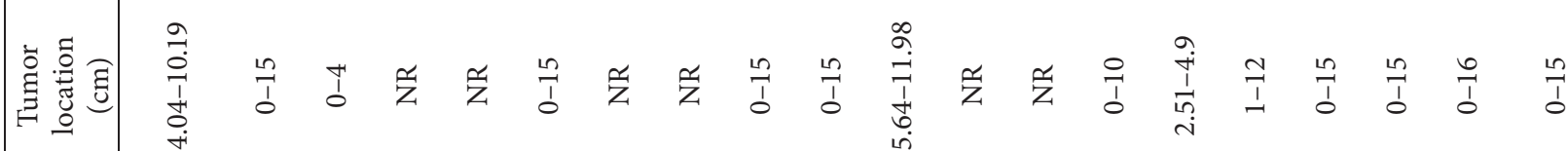

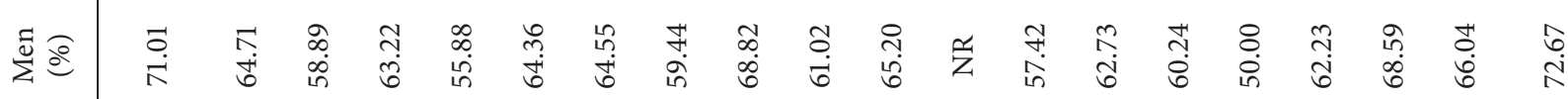

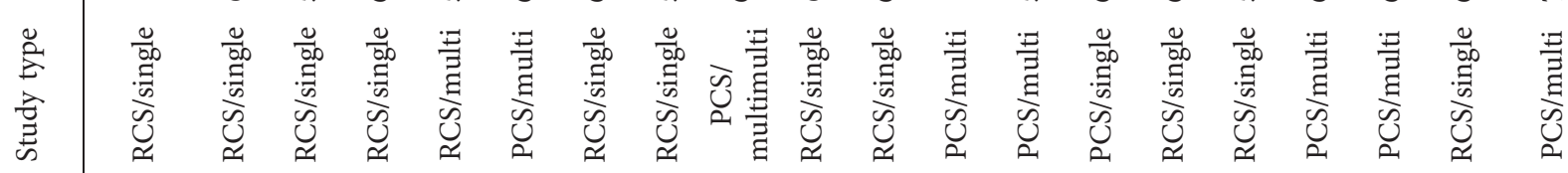

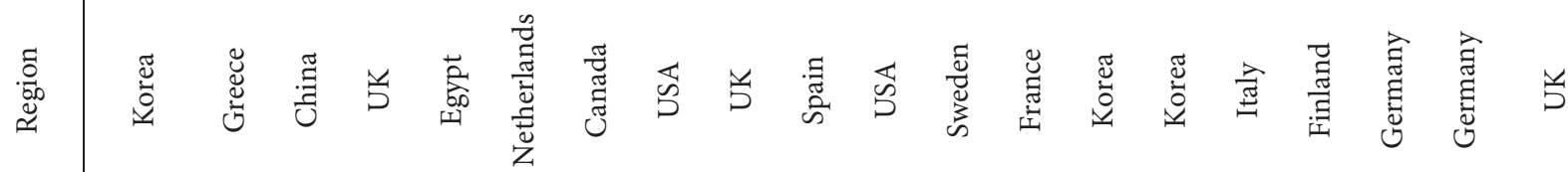

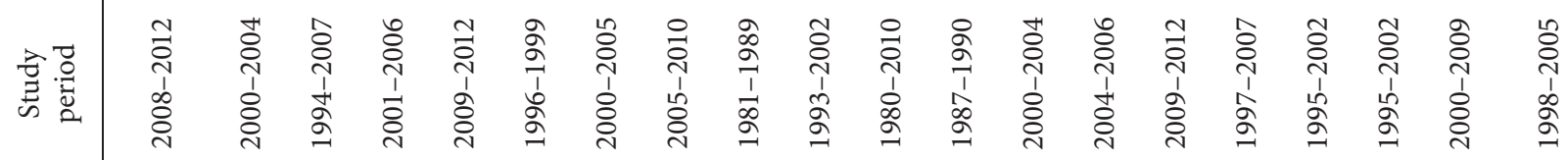

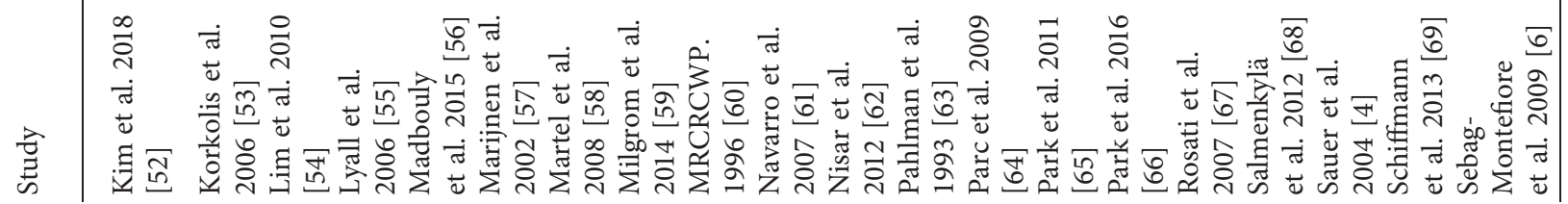




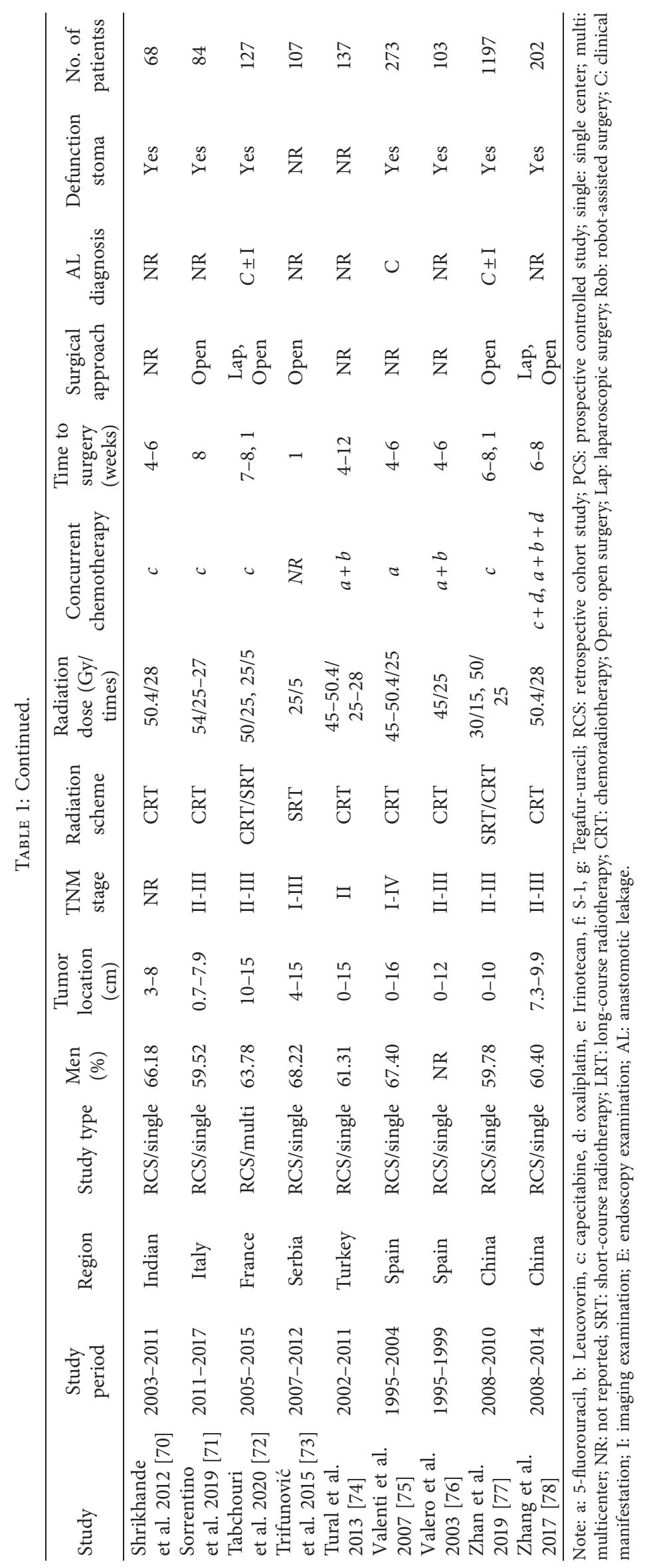




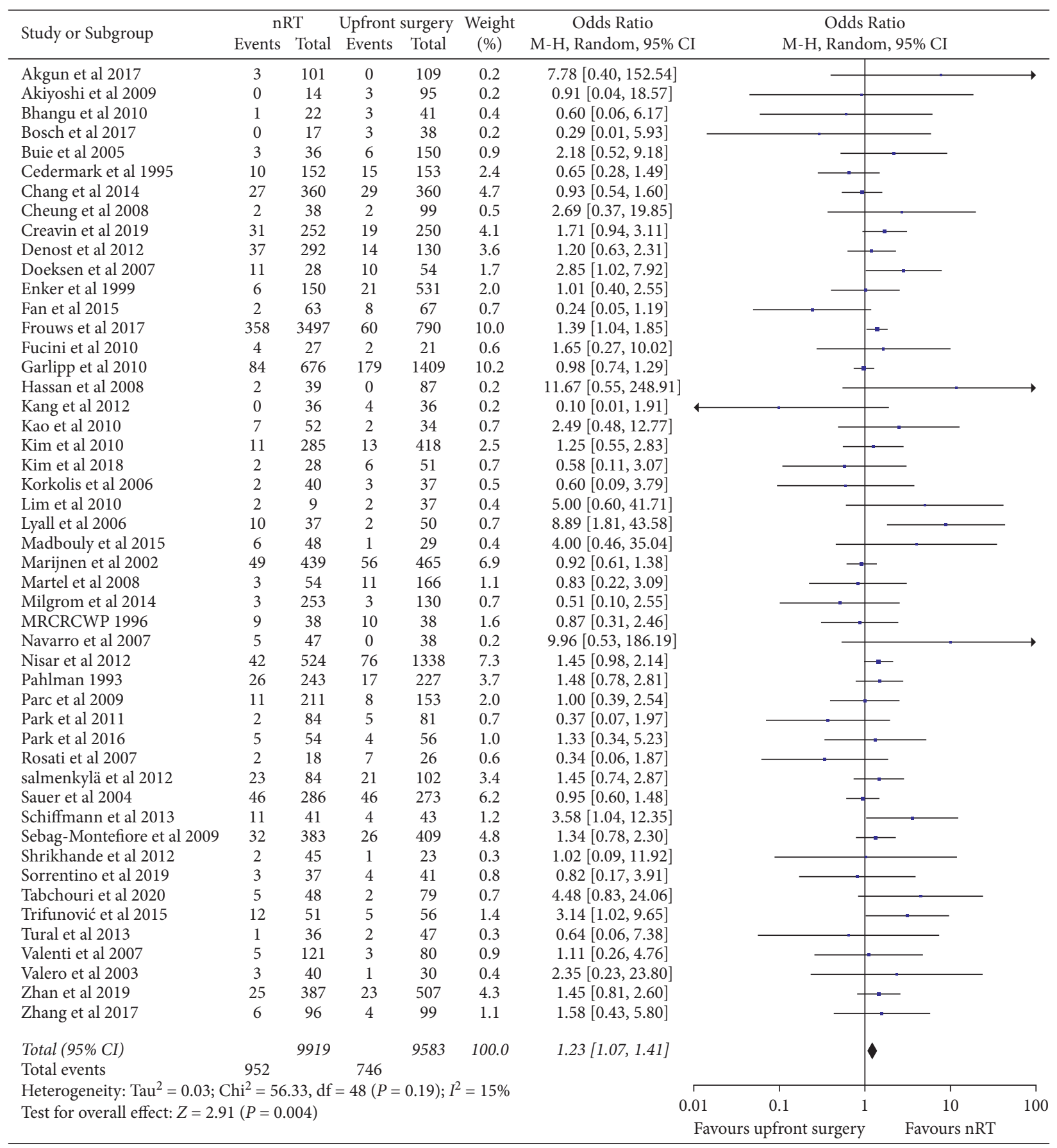

FIGURE 2: Impact of neoadjuvant radiotherapy on anastomotic leakage.

weeks after radiotherapy has been considered the optimal time for surgery. The results of two previous two metaanalyses have also shown that extending the interval of radiotherapy and surgery to 8 weeks could achieve a higher PCR rate without increasing postoperative complications $[81,82]$. Nevertheless, the GRECCAR-6 study found that prolonged operation interval after the end of radiotherapy does not improve the PCR rate and also causes more postoperative complications and surgery time [83]. A possible reason might be that the aggravation of local tissue fibrosis, edema, and inflammatory reaction in the pelvic radiation area change the normal anatomical plane, making it difficult to achieve standard TME surgery and R0 resection. The relationship between PCR and postoperative complications is a debated topic. Maggiori et al. [84] reported that the risk of $\mathrm{AL}$ and serious complications is significantly decreased in rectal cancer patients with radiotherapy sensitivity. Another study has also found that patients with PCR have a lower incidence of AL and organ space surgical site infection than patients with non-PCR [85]. In contrast to earlier findings, however, a populationbased study has suggested that rectal cancer patients who 


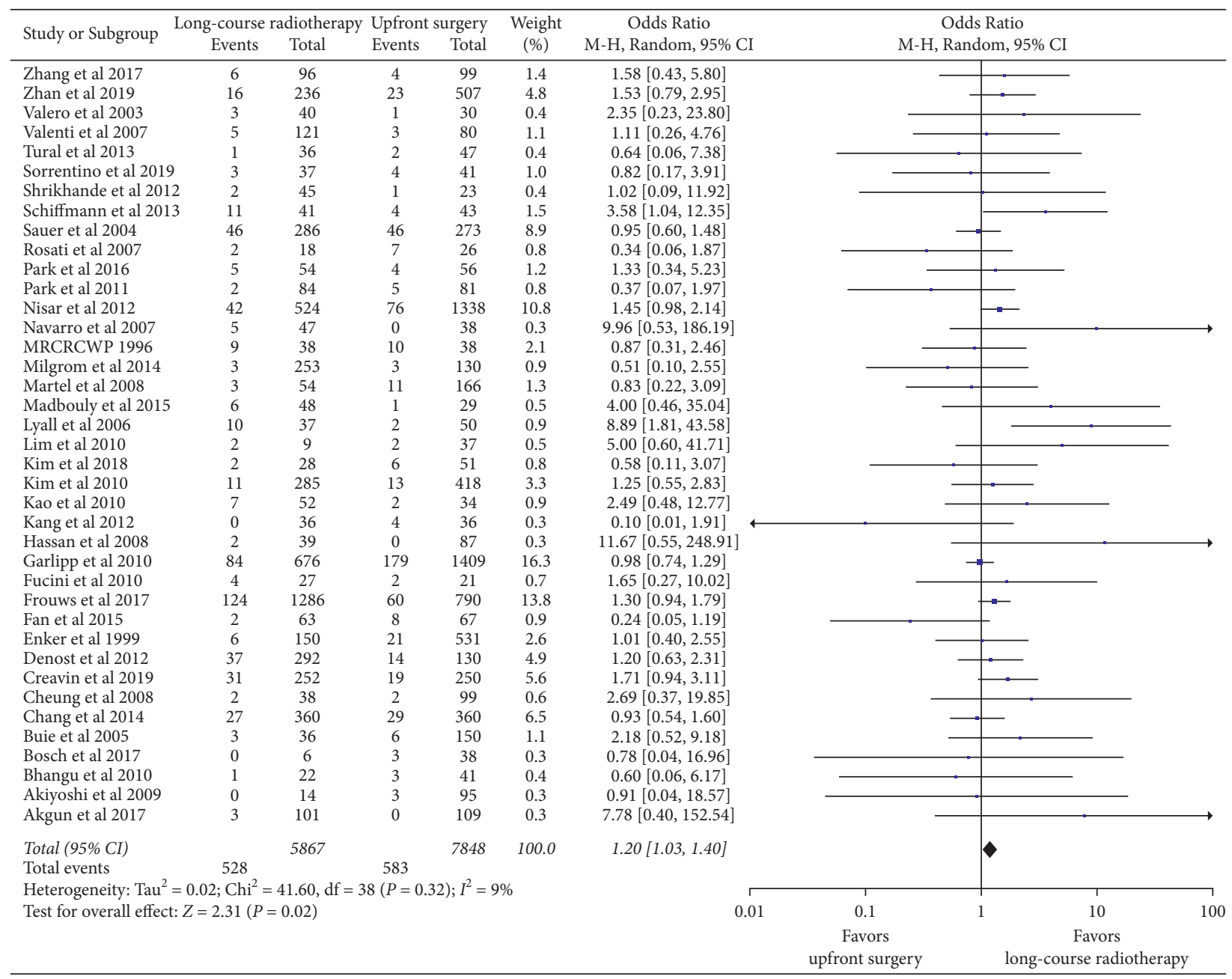

FIGURE 3: Subgroup analysis: the effect of neoadjuvant long-course radiotherapy on anastomotic leakage.

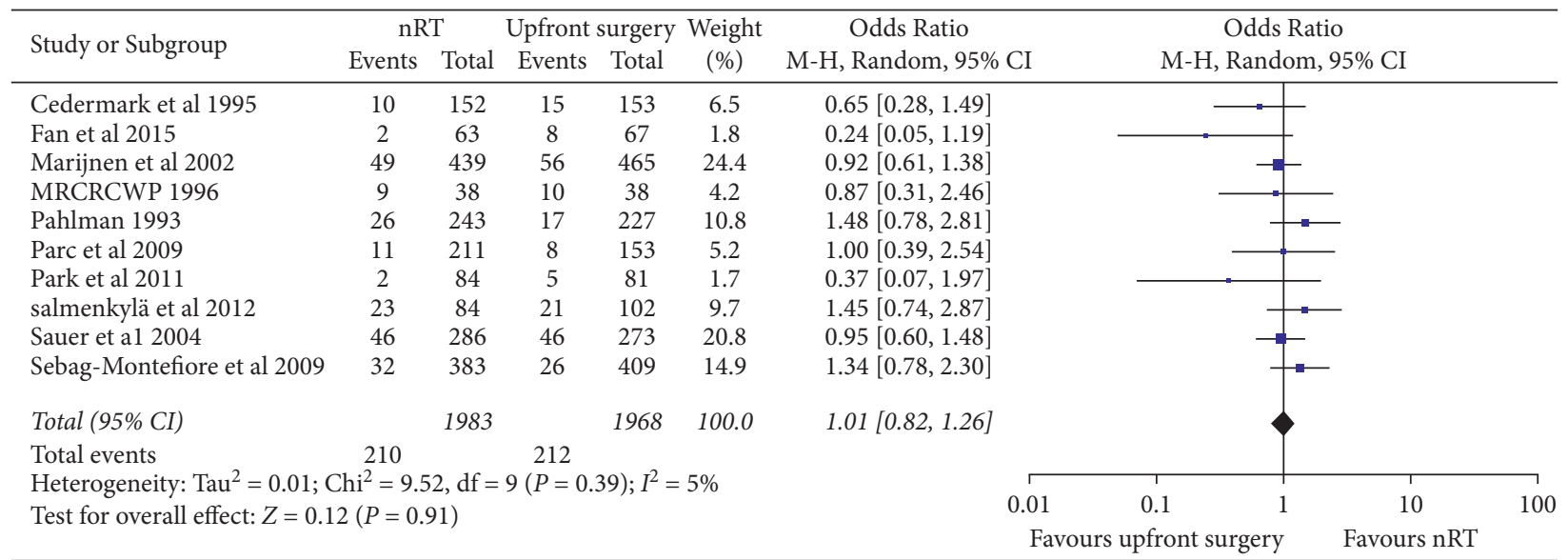

FIgURE 4: Forest plots of anastomotic leakage after neoadjuvant radiotherapy in randomized controlled trials.

achieve PCR have higher AL, surgical complications, and all complications [86].

The possible explanation for AL caused by radiotherapy might be that pelvic radiation increases the inflammatory response of the rectum and anastomosis and promotes cicatrization, which affects the healing of the anastomosis [20]. Radiation could also impel fibrosis of the intestinal connective tissue, thereby decreasing the firmness of the anastomosis $[87,88]$. In addition, radiotherapy may result in vascular occlusion of the gut and a decrease in vascular 


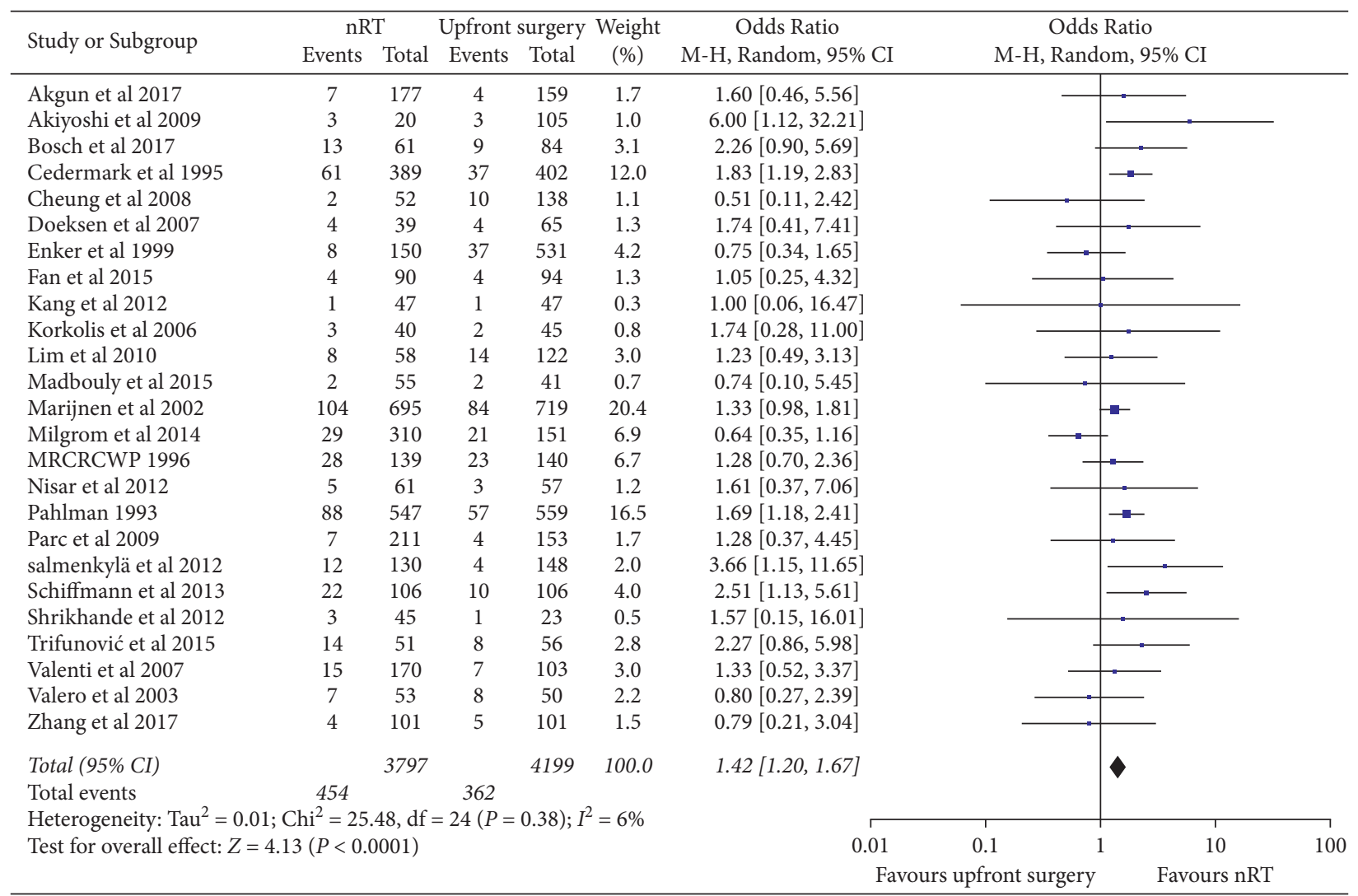

FIGURE 5: Forest plot of postoperative wound infection in patients with neoadjuvant radiotherapy.

density, which hinders the blood supply and venous reflux of the anastomosis $[89,90]$. Tissue edema and fibrosis in radiotherapy areas also challenge the surgeon's technique.

It has been reported that defunctioning stoma can decrease the risk of AL and the serious consequences after AL [91-93]. Two meta-analyses have shown that the incidence of $\mathrm{AL}$ and reoperation rates in patients with defunctioning stoma are lower than those in patients without a defunctioning stoma [94, 95]. Another meta-analysis involving 23 observational studies further concluded that defunctioning stoma is an important protective factor for AL after low anterior resection [96]. Unfortunately, several retrospective studies have demonstrated that defunctioning stoma does not impact the occurrence of AL but instead decreases the severe clinical symptoms and reoperation rate after $\mathrm{AL}$ [97-101]. In the present study, the pooled data from 21 studies showed that the defunctioning stoma rate in the $\mathrm{nRT}$ group was significantly higher than that in the upfront surgery group (69.44\% vs. $47.39 \%)$. Hence, we hypothesized that defunctioning stoma may decrease the occurrence of symptomatic AL after nRT. The main factors of AL after anterior resection are poor blood supply, excessive tension, and poor local condition of the intestine at the anastomosis. Defunctioning stoma directly reduces the pressure in the rectum and the defecation reflex caused by the stimulation of intestinal contents, thus preventing the occurrence of AL. It should be noted that defunctioning stoma did not decrease the risk of AL related to the poor anastomosis blood supply.
The present study also confirmed that nRT was associated with surgical site infection. The Stockholm Phase I trial randomly divided patients into the preoperative radiation therapy group $(5 \times 5 \mathrm{~Gy})$ and surgery alone group [38]. The results indicated that the risk of wound infection in patients with radiotherapy was higher than that of patients with surgery alone (14\% vs. $9 \%)$. Three other prospective studies also reported that SRT not only increased the occurrence of wound infection, but was also a risk factor for perineal wound infection and pelvic abscess after APR [6, 57, 63]. Consistent with SRT, CRT also plays an important role in surgical site infection after rectal cancer surgery. El-Gazzaz et al. conducted a retrospective study of 696 patients undergoing APR [102]. The data showed that, compared to surgery alone, patients receiving CRT had a higher percentage of perineal wound infections (18\% vs. 11\%). A previous meta-analysis has also found that CRT is closely related to wound infection, perineal wound infection, and AL [103]. The possible reasons are that radiation not only damages tumor cells, but also affects the peripheral microcirculation, resulting in obstructive vasculitis and delayed wound healing.

The present meta-analysis may have several limitations. First, most of the included studies were retrospective studies with a wide publication time range. Second, due to the limited number of included studies, some subgroup analysis conclusions may not be reliable. Third, the incidence of symptomatic $\mathrm{AL}$ in this meta-analysis ranged from $0 \%$ to 


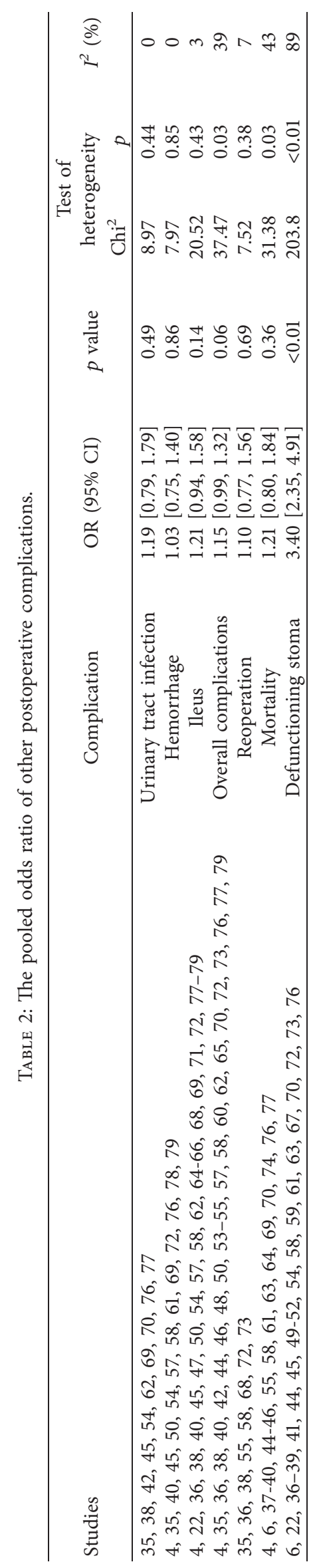


$39.29 \%$. This large difference in the AL rate may be due to the inconsistent definition of $\mathrm{AL}$ and the proportion of patients undergoing anterior rectal resection. Moreover, the conclusion that $\mathrm{nRT}$ increases the AL rate must be interpreted carefully. The pooled data in the RCTs showed that patients receiving $\mathrm{nRT}$ did not observe a higher AL rate than patients receiving upfront surgery. Fifth, we excluded 11 non-English articles. Although we found that it had no significant impact on the results after including non-English studies, it might also increase the risk of selection bias and reduce reliability. Finally, the chemotherapy regimens were different in the included CRT studies. Studies have reported that different chemotherapy regimens are associated with postoperative complications [104, 105]. However, the present metaanalysis did not conduct a subgroup analysis of the relationship between different chemotherapy regimens and postoperative complications. Hence, more randomized controlled studies are needed to further confirm these results in the future.

\section{Conclusions}

In conclusion, the present study investigated the relationship between nRT and postoperative complications. The metaanalysis suggested that nRT may increase the risk of AL, wound infection, and pelvic abscess compared to upfront surgery among patients with rectal cancer. Moreover, nRT did not significantly affect urinary tract infection, hemorrhage, ileus, reoperation, or mortality.

\section{Data Availability}

All data generated or analyzed during this study are included in this article. Further inquiries can be directed to the corresponding author.

\section{Consent}

Not applicable.

\section{Conflicts of Interest}

The authors declare no conflicts of interest.

\section{Authors' Contributions}

Conception and design were done by ZF, YJ; literature retrieval was done by YJ, YL; extraction and summary of data were done by TT, DP, YL, and YJ; statistical analysis was done by YJ, TT; drafting of the manuscript was done by YJ, DP; critical revision of the manuscript was done by ZF, DP, YJ, TT, and YL; study supervision was done by ZF. All listed authors have contributed substantially to the design, data collection and analysis, and editing of the manuscript.

\section{Acknowledgments}

This work was supported by Chongqing Key Disease Research and Application Demonstration Program (Colorectal Cancer Prevention and Treatment Technology Research and
Application Demonstration (No. 2019ZX003)) and National Natural Science Foundation of China (Grant no. 81572319).

\section{Supplementary Materials}

Supplementary Table 1. The PRISMA 2020 checklist. Supplementary Table 2. Details of postoperative complications in the neoadjuvant radiotherapy and upfront surgery group. Supplementary Table 3. The Quality assessment of cohort studies was based on the Newcastle-Ottawa scale. Supplementary Figure 1. The effect of neoadjuvant short-course radiotherapy on anastomotic leakage. Supplementary Figure 2. Forest plots of anastomotic leakage after neoadjuvant radiotherapy in cohort studies. Supplementary Figure 3. Impact of the surgery within 8 weeks after long-course radiotherapy on anastomotic leakage. Supplementary Figure 4. Forest plot of perineal wound infection after neoadjuvant radiotherapy. Supplementary Figure 5. Forest plot of pelvic abscess after neoadjuvant radiotherapy. Supplementary Figure 6. Funnel plot for anastomotic leakage. Supplementary Figure 7. Summary of risk of bias judgements for RCTs. (Supplementary Materials)

\section{References}

[1] F. Bray, J. Ferlay, I. Soerjomataram, R. L. Siegel, L. A. Torre, and A. Jemal, "Global cancer statistics 2018: GLOBOCAN estimates of incidence and mortality worldwide for 36 cancers in 185 countries," CA: A Cancer Journal for Clinicians, vol. 68, no. 6, pp. 394-424, 2018.

[2] K. C. M. J. Peeters, C. A. M. Marijnen, I. D. Nagtegaal et al., "The TME trial after a median follow-up of 6 years," Annals of Surgery, vol. 246, no. 5, pp. 693-701, 2007.

[3] R. J. Heald and R. D. H. Ryall, "Recurrence and survival after total mesorectal excision for rectal cancer," The Lancet, vol. 327, no. 8496, pp. 1479-1482, 1986.

[4] R. Sauer, T. Liersch, S. Merkel et al., "Preoperative versus postoperative chemoradiotherapy for locally advanced rectal cancer: results of the German CAO/ARO/AIO-94 randomized phase III trial after a median follow-up of 11 years," Journal of Clinical Oncology, vol. 30, no. 16, pp. 1926-1933, 2012.

[5] J.-F. Bosset, L. Collette, G. Calais et al., "Chemotherapy with preoperative radiotherapy in rectal cancer," New England Journal of Medicine, vol. 355, no. 11, pp. 1114-1123, 2006.

[6] D. Sebag-Montefiore, R. J. Stephens, R. Steele et al., "Preoperative radiotherapy versus selective postoperative chemoradiotherapy in patients with rectal cancer (MRC CR07 and NCIC-CTG C016): a multicentre, randomised trial," The Lancet, vol. 373, no. 9666, pp. 811-820, 2009.

[7] W. van Gijn, C. A. Marijnen, I. D. Nagtegaal et al., "Preoperative radiotherapy combined with total mesorectal excision for resectable rectal cancer: 12-year follow-up of the multicentre, randomised controlled TME trial," The Lancet Oncology, vol. 12, no. 6, pp. 575-582, 2011.

[8] M. Maas, P. J. Nelemans, V. Valentini et al., "Long-term outcome in patients with a pathological complete response after chemoradiation for rectal cancer: a pooled analysis of individual patient data," The Lancet Oncology, vol. 11, no. 9, pp. 835-844, 2010. 
[9] A. Kasi, S. Abbasi, S. Handa et al., "Total neoadjuvant therapy vs standard therapy in locally advanced rectal cancer," JAMA Network Open, vol. 3, no. 12, p. e2030097, 2020.

[10] M. Omejc and M. Potisek, "Prognostic significance of tumor regression in locally advanced rectal cancer after preoperative radiochemotherapy," Radiology and Oncology, vol. 52, no. 1, pp. 30-35, 2017.

[11] A. C. Gamboa, R. M. Lee, M. K. Turgeon et al., "Impact of postoperative complications on oncologic outcomes after rectal cancer surgery: an analysis of the US rectal cancer consortium," Annals of Surgical Oncology, vol. 28, no. 3, pp. 1712-1721, 2021.

[12] W. Ramphal, J. R. E. Boeding, P. D. Gobardhan et al., "Oncologic outcome and recurrence rate following anastomotic leakage after curative resection for colorectal cancer," Surgical Oncology, vol. 27, no. 4, pp. 730-736, 2018.

[13] A. Artus, N. Tabchouri, O. Iskander et al., "Long term outcome of anastomotic leakage in patients undergoing low anterior resection for rectal cancer," BMC Cancer, vol. 20, no. 1, p. $780,2020$.

[14] D. Van de Putte, C. Demarquay, E. Van Daele et al., "Adipose-derived mesenchymal stromal cells improve the healing of colonic anastomoses following high dose of irradiation through anti-inflammatory and angiogenic processes," Cell Transplantation, vol. 26, no. 12, pp. 1919-1930, 2017.

[15] M. E. Allaix, F. Rebecchi, F. Famiglietti, S. Arolfo, A. Arezzo, and M. Morino, "Long-term oncologic outcomes following anastomotic leak after anterior resection for rectal cancer: does the leak severity matter?" Surgical Endoscopy, vol. 34, no. 9, pp. 4166-4176, 2020.

[16] J. Lawler, M. Choynowski, K. Bailey, M. Bucholc, A. Johnston, and M. Sugrue, "Meta-analysis of the impact of postoperative infective complications on oncological outcomes in colorectal cancer surgery," BJS Open, vol. 4, no. 5, pp. 737-747, 2020.

[17] S. Wang, J. Liu, S. Wang, H. Zhao, S. Ge, and W. Wang, "Adverse effects of anastomotic leakage on local recurrence and survival after curative anterior resection for rectal cancer: a systematic review and meta-analysis," World Journal of Surgery, vol. 41, no. 1, pp. 277-284, 2017.

[18] J. Yang, Q. Chen, L. Jindou, and Y. Cheng, "The influence of anastomotic leakage for rectal cancer oncologic outcome: a systematic review and meta-analysis," Journal of Surgical Oncology, vol. 121, no. 8, pp. 1283-1297, 2020.

[19] J. S. Park, G.-S. Choi, S. H. Kim et al., "Multicenter analysis of risk factors for anastomotic leakage after laparoscopic rectal cancer excision," Annals of Surgery, vol. 257, no. 4, pp. 665-671, 2013.

[20] M. Yasui, I. Takemasa, Y. Miyake et al., "Tumor size as an independent risk factor for postoperative complications in laparoscopic low anterior resection for advanced rectal cancer: a multicenter Japanese study," Surgical Laparoscopy Endoscopy \& Percutaneous Techniques, vol. 27, no. 2, pp. 98-103, 2017.

[21] H. Qu, Y. Liu, and D.-S. Bi, "Clinical risk factors for anastomotic leakage after laparoscopic anterior resection for rectal cancer: a systematic review and meta-analysis," Surgical Endoscopy, vol. 29, no. 12, pp. 3608-3617, 2015.

[22] I. Hassan, D. W. Larson, B. G. Wolff et al., "Impact of pelvic radiotherapy on morbidity and durability of sphincter preservation after coloanal anastomosis for rectal cancers," Diseases of the Colon \& Rectum, vol. 51, no. 1, pp. 32-37, 2008.
[23] N. Leupin, J. Curschmann, H. Kranzbühler, C. A. Maurer, J. A. Laissue, and L. Mazzucchelli, "Acute radiation colitis in patients treated with short-term preoperative radiotherapy for rectal cancer," The American Journal of Surgical Pathology, vol. 26, no. 4, pp. 498-504, 2002.

[24] Q. Qin, Y. Zhu, P. Wu et al., "Radiation-induced injury on surgical margins: a clue to anastomotic leakage after rectalcancer resection with neoadjuvant chemoradiotherapy?" Gastroenterology Report, vol. 7, no. 2, pp. 98-106, 2019.

[25] M. J. Page, J. E. McKenzie, P. M. Bossuyt et al., "The PRISMA 2020 statement: an updated guideline for reporting systematic reviews," Journal of Clinical Epidemiology, vol. 134, pp. 178-189, 2021.

[26] G. Wells, B. Shea, D. O'Connell et al.: The newcastle-ottawa Scale (NOS) for assessing the quality of nonrandomised studies in meta-analyses, http://www.ohri.ca/programs/ clinical_epidemiology/oxford.asp.

[27] J. A. C. Sterne, J. Savović, and M. J. Page, "RoB 2: a revised tool for assessing risk of bias in randomised trials," $B M J$, vol. 366, p. 14898, 2019.

[28] K. Trencheva, K. P. Morrissey, M. Wells et al., "Identifying important predictors for anastomotic leak after colon and rectal resection," Annals of Surgery, vol. 257, no. 1, pp. 108-113, 2013.

[29] J. P. T. Higgins and S. Green, Cochrane Handbook for Systematic Reviews of Interventions, Cochrane, London, UK, 2019.

[30] R. DerSimonian and N. Laird, "Meta-analysis in clinical trials revisited," Contemporary Clinical Trials, vol. 45, no. Pt A, pp. 139-145, 2015.

[31] X. Sun, M. Briel, S. D. Walter, and G. H. Guyatt, "Is a subgroup effect believable? Updating criteria to evaluate the credibility of subgroup analyses," BMJ, vol. 340, no. 3, p. c117, 2010.

[32] L. Lin, "Graphical augmentations to sample-size-based funnel plot in meta-analysis," Research Synthesis Methods, vol. 10, no. 3, pp. 376-388, 2019.

[33] E. Akgun, S. Ozkok, M. Tekin et al., "The effects of chemoradiotherapy on recurrence and survival in locally advanced rectal cancers with curative total mesorectal excision: a prospective, nonrandomized study," World Journal of Surgical Oncology, vol. 15, no. 1, p. 205, 2017.

[34] T. Akiyoshi, H. Kuroyanagi, M. Oya et al., "Safety of laparoscopic total mesorectal excision for low rectal cancer with preoperative chemoradiation therapy," Journal of Gastrointestinal Surgery, vol. 13, no. 3, pp. 521-525, 2009.

[35] A. Bhangu, P. Nightingale, D. Daniels, and R. Tiramula, "Neoadjuvant chemoradiotherapy, sepsis and neutrophil levels following radical excision of rectal cancer," International Journal of Colorectal Disease, vol. 25, no. 6, pp. 723729, 2010

[36] S. L. Bosch, S. J. van Rooijen, G. M. J. Bökkerink et al., “Acute toxicity and surgical complications after preoperative (chemo) radiation therapy for rectal cancer in patients with inflammatory bowel disease," Radiotherapy \& Oncology, vol. 123, no. 1, pp. 147-153, 2017.

[37] D. W. Buie, A. R. MacLean, J.-A. P. Attard, P. M. A. Brasher, and A. K. Chan, "Neoadjuvant chemoradiation increases the risk of pelvic sepsis after radical excision of rectal cancer," Diseases of the Colon \& Rectum, vol. 48, no. 10, pp. 18681874, 2005.

[38] B. Cedermark, H. Johansson, L. E. Rutqvist, and N. Wilking, "The Stockholm I trial of preoperative short term 
radiotherapy in operable rectal carcinoma," Stockholm Colorectal Cancer Study Group, vol. 75, no. 9, pp. 2269-2275, 1995.

[39] J. S. Chang, K. C. Keum, N. K. Kim et al., "Preoperative chemoradiotherapy effects on anastomotic leakage after rectal cancer resection," Annals of Surgery, vol. 259, no. 3, pp. 516-521, 2014.

[40] H. Y. S. Cheung, C. C. Chung, J. C. H. Wong, K. K. K. Yau, and M. K. W. Li, "Laparoscopic rectal cancer surgery with and without neoadjuvant chemo-irradiation: a comparative study," Surgical Endoscopy, vol. 23, no. 1, pp. 147-152, 2009.

[41] B. Creavin, É. J. Ryan, M. E. Kelly et al., "Minimally invasive approaches to the management of anastomotic leakage following restorative rectal cancer resection," Colorectal Disease, vol. 21, no. 12, pp. 1364-1371, 2019.

[42] Q. Denost, C. Laurent, T. Paumet, L. Quintane, M. Martenot, and E. Rullier, "Laparoscopic surgery for rectal cancer: preoperative radiochemotherapy versus surgery alone," Surgical Endoscopy, vol. 26, no. 7, pp. 1878-1883, 2012.

[43] A. Doeksen, P. J. Tanis, B. C. Vrouenraets, J. A Gooszen, J. J van Lanschot, and W. F van Tets, "Outcome of rectal cancer surgery after the introduction of preoperative radiotherapy in a low-volume hospital," Journal of Gastrointestinal Cancer, vol. 38, no. 2-4, pp. 63-70, 2007.

[44] W. E. Enker, N. Merchant, A. M. Cohen et al., "Safety and efficacy of low anterior resection for rectal cancer," Annals of Surgery, vol. 230, no. 4, pp. 544-554, 1999.

[45] W. H. Fan, F. L. Wang, and Z. H. Lu, "Surgery with versus without preoperative concurrent chemoradiotherapy for $\mathrm{mid} /$ low rectal cancer: an interim analysis of a prospective, randomized trial," Chinese Journal of Cancer, vol. 34, no. 9, pp. 394-403, 2015.

[46] M. A. Frouws, H. S. Snijders, S. H. Malm et al., "Clinical relevance of a grading system for anastomotic leakage after low anterior resection: analysis from a national cohort database," Diseases of the Colon \& Rectum, vol. 60, no. 7, pp. 706-713, 2017.

[47] C. Fucini, F. Pucciani, C. Elbetti, R. Gattai, and A. Russo, "Preoperative radiochemotherapy in $\mathrm{t} 3$ operable low rectal cancers: a gold standard?" World Journal of Surgery, vol. 34, no. 7, pp. 1609-1614, 2010.

[48] B. Garlipp, H. Ptok, U. Schmidt, F. Meyer, I. Gastinger, and H. Lippert, "Neoadjuvant chemoradiotherapy for rectal carcinoma: effects on anastomotic leak rate and postoperative bladder dysfunction after non-emergency sphincterpreserving anterior rectal resection," Langenbeck's Archives of Surgery, vol. 395, no. 8, pp. 1031-1038, 2010.

[49] J. Kang, S. M. Jang, J.-H. Baek, W. S. Lee, and T. H. Cho, "Short-term results and long-term oncologic outcomes between neoadjuvant chemoradiotherapy and adjuvant postoperative chemoradiotherapy for stage III rectal cancer: a case-matched study," Annals of Surgical Oncology, vol. 19, no. 8, pp. 2494-2499, 2012.

[50] P.-S. Kao, S.-C. Chang, L.-W. Wang et al., "The impact of preoperative chemoradiotherapy on advanced low rectal cancer," Journal of Surgical Oncology, vol. 102, no. 7, pp. 771-777, 2010.

[51] C. W. Kim, J. H. Kim, C. S. Yu et al., "Complications after sphincter-saving resection in rectal cancer patients according to whether chemoradiotherapy is performed before or after surgery," International Journal of Radiation Oncology, Biology, Physics, vol. 78, no. 1, pp. 156-163, 2010.

[52] J. G. Kim, K. D. Song, D. I. Cha, H. C. Kim, and J. I. Yu, "Indistinguishable T2/T3-N0 rectal cancer on rectal magnetic resonance imaging: comparison of surgery-first and neoadjuvant chemoradiation therapy-first strategies," International Journal of Colorectal Disease, vol. 33, no. 10, pp. 1359-1366, 2018.

[53] D. P. Korkolis, G. D. Plataniotis, E. Gondikakis et al., "Shortterm preoperative radiotherapy is a safe approach for treatment of locally advanced rectal cancer," International Journal of Colorectal Disease, vol. 21, no. 1, pp. 1-6, 2006.

[54] Y. K. Lim, W. L. Law, and R. Liu, "Impact of neoadjuvant treatment on total mesorectal excision for ultra-low rectal cancers," World Journal of Surgical Oncology, vol. 8, no. 23, p. 23, 2010.

[55] A. Lyall, T. K. Mc Adam, J. Townend, and M. A. Loudon, "Factors affecting anastomotic complications following anterior resection in rectal cancer," Colorectal Disease, vol. 9, no. 9, pp. 801-807, 2007.

[56] K. M. Madbouly, A. N. Mashhour, and W. Omar, "Is it safe to omit neoadjuvant chemo-radiation in mucinous rectal carcinoma?" International Journal of Surgery, vol. 23, no. Pt A, pp. 120-127, 2015.

[57] C. A. M. Marijnen, E. Kapiteijn, C. J. H. Van De Velde et al., "Acute side effects and complications after short-term preoperative radiotherapy combined with total mesorectal excision in primary rectal cancer: report of a multicenter randomized trial," Journal of Clinical Oncology, vol. 20, no. 3, pp. 817-825, 2002.

[58] G. Martel, Y. Al-Suhaibani, H. Moloo et al., "Neoadjuvant therapy and anastomotic leak after tumor-specific mesorectal excision for rectal cancer," Diseases of the Colon \& Rectum, vol. 51, no. 8, pp. 1195-1201, 2008.

[59] S. A. Milgrom, K. A. Goodman, G. M. Nash et al., "Neoadjuvant radiation therapy prior to total mesorectal excision for rectal cancer is not associated with postoperative complications using current techniques," Annals of Surgical Oncology, vol. 21, no. 7, pp. 2295-2302, 2014.

[60] Medical Research Council Rectal Cancer Working Party, "Randomised trial of surgery alone versus radiotherapy followed by surgery for potentially operable locally advanced rectal cancer," Lancet, vol. 348, no. 9042, pp. 1605-1610, 1996.

[61] G. Valero Navarro, J. A. Luján Mompeán, Q. Hernández Agüera et al., "Influence of the neo-adjuvant radiochemotherapy as a factor in the surgical treatment of rectal cancer by expert surgeon. A comparative study," International Journal of Colorectal Disease, vol. 22, no. 10, pp. 1233-1238, 2007.

[62] P. J. Nisar, I. C. Lavery, and R. P. Kiran, "Influence of neoadjuvant radiotherapy on anastomotic leak after restorative resection for rectal cancer," Journal of Gastrointestinal Surgery, vol. 16, no. 9, pp. 1750-1757, 2012.

[63] Swedish Rectal Cancer Trial, "Initial report from a Swedish multicentre study examining the role of preoperative irradiation in the treatment of patients with resectable rectal carcinoma," British Journal of Surgery, vol. 80, no. 10, pp. 1333-1336, 1993.

[64] Y. Parc, M. Zutshi, S. Zalinski, R. Ruppert, A. Fürst, and V. W. Fazio, "Preoperative radiotherapy is associated with worse functional results after coloanal anastomosis for rectal cancer," Diseases of the Colon \& Rectum, vol. 52, no. 12, pp. 2004-2014, 2009.

[65] J.-h. Park, S. M. Yoon, C. S. Yu, J. H. Kim, T. W. Kim, and J. C. Kim, "Randomized phase 3 trial comparing preoperative and postoperative chemoradiotherapy with capecitabine for 
locally advanced rectal cancer," Cancer, vol. 117, no. 16, pp. 3703-3712, 2011.

[66] I. J. Park, C. S. Yu, S.-B. Lim et al., "Is preoperative chemoradiotherapy beneficial for sphincter preservation in lowlying rectal cancer patients?" Medicine, vol. 95, no. 18, p. e3463, 2016.

[67] R. Rosati, S. Bona, and U. F. Romario, "Laparoscopic total mesorectal excision after neoadjuvant chemoradiotherapy," Surg Oncol, vol. 16, no. 1, pp. S83-S89, 2007.

[68] S. Salmenkylä, M. Kouri, and P. Österlund, "Does preoperative radiotherapy with postoperative chemotherapy increase acute side-effects and postoperative complications of total mesorectal excision? Report of the randomized Finnish rectal cancer trial," Scandinavian Journal of Surgery, vol. 101, no. 4, pp. 275-282, 2012.

[69] L. Schiffmann, N. Wedermann, M. Gock et al., "Intensified neoadjuvant radiochemotherapy for rectal cancer enhances surgical complications," BMC Surgery, vol. 13, no. 1, p. 43, 2013.

[70] S. V. Shrikhande, Y. D. Bodhankar, K. Suradkar, M. Goel, and P. J. Shukla, "Perioperative outcomes after ultra low anterior resection in the era of neoadjuvant chemoradiotherapy," Indian Journal of Gastroenterology, vol. 32, no. 2, pp. 90-97, 2013.

[71] L. Sorrentino, M. Guaglio, L. Battaglia et al., "Neoadjuvant chemo-radiotherapy for cT3N0 rectal cancer: any benefit over upfront surgery? A propensity score-matched study," International Journal of Colorectal Disease, vol. 34, no. 12, pp. 2161-2169, 2019.

[72] N. Tabchouri, Y. Eid, G. Manceau et al., "Neoadjuvant treatment in upper rectal cancer does not improve oncologic outcomes but increases postoperative morbidity," Anticancer Research, vol. 40, no. 6, pp. 3579-3587, 2020.

[73] B. Trifunovic, J. Krsic, M. Bezmarevic et al., "Relationship of short-course preoperative radiotherapy and serum albumin level with postoperative complications in rectal cancer surgery," Vojnosanitetski Pregled, vol. 72, no. 8, pp. 663-669, 2015.

[74] D. Tural, F. Selcukbiricik, Ö. Yıldız et al., "Preoperative versus postoperative chemoradiotherapy in stage T3, N0 rectal cancer," International Journal of Clinical Oncology, vol. 19, no. 5, pp. 889-896, 2014.

[75] V. Valenti, J. L. Hernandez-Lizoain, J. Baixauli et al., "Analysis of early postoperative morbidity among patients with rectal cancer treated with and without neoadjuvant chemoradiotherapy," Annals of Surgical Oncology, vol. 14, no. 5, pp. 1744-1751, 2007.

[76] G. Valero, J. A. Luján, Q. Hernández et al., "Neoadjuvant radiation and chemotherapy in rectal cancer does not increase postoperative complications," International Journal of Colorectal Disease, vol. 18, no. 6, pp. 495-499, 2003.

[77] T.-C. Zhan, D.-K. Zhang, J. Gu, and M. Li, "Surgical complications after different therapeutic approaches for locally advanced rectal cancer," World Journal of Gastrointestinal Oncology, vol. 11, no. 5, pp. 393-403, 2019.

[78] Y. Zhang, Y. Sun, Z. Xu, P. Chi, and X. Lu, "Is neoadjuvant chemoradiotherapy always necessary for mid/high local advanced rectal cancer: a comparative analysis after propensity score matching," European Journal of Surgical Oncology, vol. 43, no. 8, pp. 1440-1446, 2017.

[79] Q. Qin, T. Ma, Y. Deng et al., "Impact of preoperative radiotherapy on anastomotic leakage and stenosis after rectal cancer resection: post hoc analysis of a randomized controlled trial," Diseases of the Colon \& Rectum, vol. 59, no. 10, pp. 934-942, 2016.

[80] Y. Francois, C. J. Nemoz, J. Baulieux et al., "Influence of the interval between preoperative radiation therapy and surgery on downstaging and on the rate of sphincter-sparing surgery for rectal cancer: the Lyon R90-01 randomized trial," Journal of Clinical Oncology, vol. 17, no. 8, p. 2396, 1999.

[81] É. J. Ryan, D. P. O’Sullivan, M. E. Kelly et al., "Meta-analysis of the effect of extending the interval after long-course chemoradiotherapy before surgery in locally advanced rectal cancer," British Journal of Surgery, vol. 106, no. 10, pp. 1298-1310, 2019.

[82] D. Du, Z. Su, D. Wang, W. Liu, and Z. Wei, “Optimal interval to surgery after neoadjuvant chemoradiotherapy in rectal cancer: a systematic review and meta-analysis," Clinical Colorectal Cancer, vol. 17, no. 1, pp. 13-24, 2018.

[83] J. H. Lefevre, L. Mineur, S. Kotti et al., "Effect of interval (7 or 11 weeks) between neoadjuvant radiochemotherapy and surgery on complete pathologic response in rectal cancer: a multicenter, randomized, controlled trial (GRECCAR-6)," Journal of Clinical Oncology, vol. 34, no. 31, pp. 3773-3780, 2016.

[84] L. Maggiori, F. Bretagnol, M. I. Aslam et al., "Does pathologic response of rectal cancer influence postoperative morbidity after neoadjuvant radiochemotherapy and total mesorectal excision?" Surgery, vol. 155, no. 3, pp. 468-475, 2014.

[85] J. H. Wolf, Y.-C. Hung, S. Cox et al., "Pathologic complete response is associated with decreased morbidity following rectal cancer resection," The American Journal of Surgery, vol. 222, no. 2, pp. 390-394, 2021.

[86] F. J. Van Der Sluis, A. M. Couwenberg, and G. H. De Bock, "Population-based study of morbidity risk associated with pathological complete response after chemoradiotherapy for rectal cancer," British Journal of Surgery, vol. 107, no. 1, pp. 131-139, 2020.

[87] J. M. Dominguez, S. M. Jakate, N. J. Speziale, M. H. Savin, W. E. Altringer, and T. J. Saclarides, "Intestinal anastomotic healing at varying times after irradiation," Journal of Surgical Research, vol. 61, no. 1, pp. 293-299, 1996.

[88] S. Jahnson, R. H. Christofferson, and B. Gerdin, "Reduced mucosal perianastomotic capillary density in rat small intestine with chronic radiation damage," Radiation Research, vol. 150 , no. 5 , pp. 542-548, 1998.

[89] N. D. Carr, B. R. Pullen, P. S. Hasleton, and P. F. Schofield, "Microvascular studies in human radiation bowel disease," Gut, vol. 25, no. 5, pp. 448-454, 1984.

[90] P. S. Hasleton, N. Carr, and P. F. Schofield, "Vascular changes in radiation bowel disease," Histopathology, vol. 9, no. 5, pp. 517-534, 1985.

[91] T. F. Eriksen, C. B. Lassen, and I. Gögenur, "Treatment with corticosteroids and the risk of anastomotic leakage following lower gastrointestinal surgery: a literature survey," Colorectal Disease, vol. 16, no. 5, pp. 154-160, 2014

[92] K. C. M. J. Peeters, R. A. E. M. Tollenaar, C. A. M. Marijnen et al., "Risk factors for anastomotic failure after total mesorectal excision of rectal cancer," British Journal of Surgery, vol. 92, no. 2, pp. 211-216, 2005.

[93] B. C. Lee, S.-B. Lim, J. L. Lee et al., "Defunctioning protective stoma can reduce the rate of anastomotic leakage after low anterior resection in rectal cancer patients," Annals of Coloproctology, vol. 36, no. 3, pp. 192-197, 2020.

[94] K. Phan, L. Oh, G. Ctercteko et al., "Does a stoma reduce the risk of anastomotic leak and need for re-operation following low anterior resection for rectal cancer: systematic review 
and meta-analysis of randomized controlled trials," Journal of Gastrointestinal Oncology, vol. 10, no. 2, pp. 179-187, 2019.

[95] N. Hüser, C. W. Michalski, and M. Erkan, "Systematic review and meta-analysis of the role of defunctioning stoma in low rectal cancer surgery," Annals of Surgery, vol. 248, no. 1, pp. 52-60, 2008.

[96] X.-T. Wang, L. Li, F.-B. Kong, X.-G. Zhong, and W. Mai, "Surgical-related risk factors associated with anastomotic leakage after resection for rectal cancer: a meta-analysis," Japanese Journal of Clinical Oncology, vol. 50, no. 1, pp. 20-28, 2020.

[97] I. Fratric, Z. Radovanovic, and D. Radovanovic, "Value of protective stoma in rectal cancer surgery," Medicinski Pregled, vol. 69, no. 3-4, pp. 73-78, 2016.

[98] K. Anderin, U. O. Gustafsson, and A. Thorell, "The effect of diverting stoma on postoperative morbidity after low anterior resection for rectal cancer in patients treated within an ERAS program," European Journal of Surgical Oncology, vol. 41, no. 6, pp. 724-730, 2016.

[99] W. E. StamR, "Impact of an institutional change from routine to highly selective diversion of a low anastomosis after TME for rectal cancer," European Journal of Surgical Oncology, vol. 44, no. 8, pp. 1220-1225, 2018.

[100] A. Shiomi, M. Ito, K. Maeda et al., "Effects of a diverting stoma on symptomatic anastomotic leakage after low anterior resection for rectal cancer: a propensity score matching analysis of 1,014 consecutive patients," Journal of the American College of Surgeons, vol. 220, no. 2, pp. 186-194, 2015.

[101] H. Shimizu, S. Yamaguchi, T. Ishii et al., "Who needs diverting ileostomy following laparoscopic low anterior resection in rectal cancer patients? Analysis of 417 patients in a single institute," Surgical Endoscopy, vol. 34, no. 2, pp. 839-846, 2020.

[102] G. El-Gazzaz, R. P. Kiran, and I. Lavery, "Wound complications in rectal cancer patients undergoing primary closure of the perineal wound after abdominoperineal resection," Diseases of the Colon \& Rectum, vol. 52, no. 12, pp. 19621966, 2009.

[103] G. D. Musters, C. J. Buskens, W. A. Bemelman, and P. J. Tanis, "Perineal wound healing after abdominoperineal resection for rectal cancer," Diseases of the Colon \& Rectum, vol. 57, no. 9, pp. 1129-1139, 2014.

[104] J. P. Gérard, D. Azria, S. Gourgou-Bourgade, I. MartelLaffay, C. Hennequin, and P. L. Etienne, "Comparison of two neoadjuvant chemoradiotherapy regimens for locally advanced rectal cancer: results of the phase III trial ACCORD 12/0405-Prodige 2," Journal of Clinical Oncology: Official Journal of the American Society of Clinical Oncology, vol. 28, no. 10, pp. 1638-1644, 2010.

[105] M. J. O’Connell, L. H. Colangelo, R. W. Beart et al., "Capecitabine and oxaliplatin in the preoperative multimodality treatment of rectal cancer: surgical end points from National Surgical Adjuvant Breast and Bowel Project trial R-04," Journal of Clinical Oncology, vol. 32, no. 18, pp. 1927-1934, 2014. 